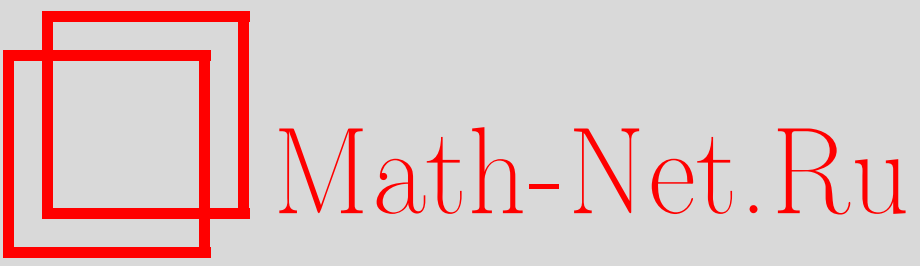

В. Г. Никонов, О строении строго выпуклых $k$-значных функций, Maтем. вопр. криптогр., 2011, том 2, выпуск 1, 75-95

DOI: https://doi.org/10.4213/mvk26

Использование Общероссийского математического портала Math-Net.Ru подразумевает, что вы прочитали и согласны с пользовательским соглашением

http://www.mathnet.ru/rus/agreement

Параметры загрузки:

IP : 54.197 .130 .99

26 апреля 2023 г., 14:32:03 
УДК 579.716.32

\section{О строении строго выпуклых $k$-значных функций 1}

\section{В. Г. Никонов}

Академия криптографии Российской Федерации, Москва

Получено 22.IV.2010

В статье изучаются строго выпуклые функции $k$-значной логики $f\left(x_{1}, \ldots, x_{n}\right), \quad x_{1}, \ldots, x_{n} \in\{0,1, \ldots, k-1\}, \quad$ для которых каждое уравнение $f\left(x_{1}, \ldots, x_{n}\right)=\alpha, \alpha \in\{0,1, \ldots, k-1\}$, можно задать равносильной системой линейных неравенств. Минимальное число неравенств $r_{\alpha}$ в такой системе называется пороговым индексом соответствующего уравнения. Для строго выпуклой функции $f\left(x_{1}, \ldots, x_{n}\right)$ вводится суммарная пороговая сложность $h=\sum_{\alpha=0}^{k-1} r_{\alpha}$ и изучаются пределы ее изменения.

Ключевые слова: функции $k$-значной логики, выпуклые функции, системы линейных неравенств

On the structure of strictly convex $k$-functions

\section{G. Nikonov}

Academy of Cryptography of Russian Federation, Moscow

Abstract. This article deals with strictly convex $k$-functions $f\left(x_{1}, \ldots, x_{n}\right)$, $x_{1}, \ldots, x_{n} \in\{0,1, \ldots, k-1\}$. For such functions each equation $f\left(x_{1}, \ldots, x_{n}\right)=\alpha$, $\alpha \in\{0,1, \ldots, k-1\}$, may be represented by an equivalent system of linear inequalities. The minimal number $r_{\alpha}$ of inequalities in the system is called the threshold index for the considering equation. For strictly convex $k$-function $f\left(x_{1}, \ldots, x_{n}\right)$ the total threshold complexity $h=\sum_{\alpha=0}^{k-1} r_{\alpha}$ is considered and the range of $h$ is investigated.

Key words: $k$-functions, convex $k$-functions, systems of linear inequalities

Citation: Mathematical Aspects of Cryptography, 2010, vol. 2, no. 1, pp. 75-96 (Russian). 
В статье [1] рассматривалась возможность задания уравнений $k$ значной логики системами линейных неравенств. Уравнение

$$
f\left(x_{1}, \ldots, x_{n}\right)=\alpha,
$$

где $x_{i}, \alpha \in\{0,1, \ldots, k-1\}, f\left(x_{1}, \ldots, x_{n}\right)$ - функция $k$-значной логики от $n$ переменных, было названо выпуклым, если подмножество точек $A_{f}^{\alpha} n$-мерной $k$-значной решетки $V_{n}^{k}=\left\{\left(\varepsilon_{1}, \ldots, \varepsilon_{n}\right) \mid \varepsilon_{i} \in\{0, \ldots, k-1\}, i=1, \ldots, n\right\}, \quad$ в которых выполняется (1), задается равносильной системой неравенств

$$
\left\{\begin{array}{c}
a_{1}^{(1)} x_{1}+\ldots+a_{n}^{(1)} x_{n} \geq b^{(1)} \\
a_{1}^{(2)} x_{1}+\ldots+a_{n}^{(2)} x_{n} \geq b^{(2)} \\
\ldots \\
a_{1}^{(r)} x_{1}+\ldots+a_{n}^{(r)} x_{n} \geq b^{(r)}
\end{array}\right.
$$

Функция $f\left(x_{1}, \ldots, x_{n}\right)$ называется строго выпуклой, если порождаемые ею уравнения вида (1) для всех $\alpha \in\{0,1, \ldots, k-1\}-$ выпуклые.

Возможность задания подмножества точек $A_{f}^{\alpha}$ системой неравенств (2) порождает задачу минимизации числа $r$. Минимальное значение $r=r_{\alpha}$ для уравнения (1) называется пороговым индексом уравнения (1).

При анализе систем уравнений

$$
f_{i}\left(x_{1}, \ldots, x_{n}\right)=\alpha_{i}, i=1, \ldots, N,
$$

порожденных строго выпуклыми функциями $f_{i}\left(x_{1}, \ldots, x_{n}\right)$, можно заменить каждое уравнение системы (3) равносильной системой неравенств и сформировать итоговую систему неравенств, равносильную системе (3) в целом. В такой замене заключается логика метода разделяющих плоскостей, который всегда применим к булевым уравнениям (см. [2, 3, 4]) и без ограничений переносится в $k$-значную область только для строго выпуклых функций.

Этим обстоятельством объясняется актуальность изучения строго выпуклых $k$-значных функций.

Важный класс строго выпуклых функций задают пороговые $k$-значные функции $f\left(x_{1}, \ldots, x_{n}\right)$ (см. [1]), для которых существует такая линейная форма $L\left(x_{1}, \ldots, x_{n}\right)=a_{1} x_{1}+\ldots+a_{n} x_{n}$, что

$$
f\left(x_{1}, \ldots, x_{n}\right)=\alpha \Leftrightarrow b_{\alpha} \leq a_{1} x_{1}+\ldots+a_{n} x_{n}<b_{\alpha+1}
$$

для любого $\alpha \in\{0,1, \ldots, k-1\}$, где $a_{i}, i=1, \ldots, n, b_{\alpha}$ - действительные числа. 
Определение 1. Суммарной пороговой сложностью $h$ строго выпуклой функции $f\left(x_{1}, \ldots, x_{n}\right)$ назовем сумму пороговых индексов всех порождаемых ею уравнений

$$
h=\sum_{\alpha=0}^{k-1} r_{\alpha} .
$$

Параметр $h$ характеризует среднее число неравенств в системе неравенств, эквивалентной порожденному данной функцией уравнению. В силу этого параметр $h$ определяет сложность записи системы неравенств в методе разделяющих плоскостей применительно к системам уравнений, порожденным функцией $f\left(x_{1}, \ldots, x_{n}\right)$.

Для пороговых $k$-значных функций суммарная пороговая сложность $h(k)$ равна

$$
h(k)=2 k-2,
$$

так как каждое уравнение задается двумя неравенствами (4), за исключением крайних, $f\left(x_{1}, \ldots, x_{n}\right)=0$ и $f\left(x_{1}, \ldots, x_{n}\right)=k-1$, которые задаются одним неравенством. В данной статье эта оценка будет отправной для изучения пределов изменения параметра $h$.

Наименьшим возможным значением суммарной пороговой сложности является $h=k$, когда каждое уравнение вида (1) представляется одним неравенством.

Теорема 1. При $k=n+1$ существуют $k$-значные функциии с суммарной пороговой сложностью $h=k$.

Доказательство теоремы состоит в рассмотрении функции специального вида $\varphi\left(x_{1}, \ldots, x_{n}\right)$, заданной следующими условиями:

$$
\begin{aligned}
& \varphi\left(x_{1}, \ldots, x_{n}\right)=0 \Leftrightarrow \Leftrightarrow x_{1}+\ldots+x_{n} \leq 0, \\
& \varphi\left(x_{1}, \ldots, x_{n}\right)=1 \quad \Leftrightarrow-x_{1}+(k-1) x_{2}+\ldots+(k-1) x_{n}<0, \\
& \varphi\left(x_{1}, \ldots, x_{n}\right)=2 \quad \Leftrightarrow-x_{2}+(k-1) x_{3}+\ldots+(k-1) x_{n}<0, \quad \ldots \quad \ldots \\
& \ldots . . \\
& \varphi\left(x_{1}, \ldots, x_{n}\right)=k-2 \Leftrightarrow-x_{n-1}+(k-1) x_{n}<0, \\
& \varphi\left(x_{1}, \ldots, x_{n}\right)=k-1 \Leftrightarrow-x_{n}<0 .
\end{aligned}
$$

Действительно, из задания функции (5) можно заключить, что неравенство с номером $\mu=0,1, \ldots, n$ имеет множество решений вида

$$
A_{\varphi}^{\mu}=\left\{\left(\xi_{1}, \ldots, \xi_{\mu-1}, \varepsilon_{\mu}, 0, \ldots, 0\right)\right\},
$$

где $\xi_{1}, \ldots, \xi_{\mu-1} \in\{0, \ldots, k-1\}-$ произвольные, а $\varepsilon_{\mu} \neq 0$. 
Отсюда вытекает, что множества решений уравнений

$$
\varphi\left(x_{1}, \ldots, x_{n}\right)=\mu_{1} \quad \text { и } \quad \varphi\left(x_{1}, \ldots, x_{n}\right)=\mu_{2}
$$

при $\mu_{1} \neq \mu_{2}$ не пересекаются: $A_{\varphi}^{\mu_{1}} \cap A_{\varphi}^{\mu_{2}}=\varnothing$, а в совокупности множества решений всех уравнений (5) покрывают все множество точек $n$-мерной $k$-значной решетки $V_{n}^{k}$

$$
\bigcup_{\mu=0}^{k-1} A_{\varphi}^{\mu_{1}}=V_{n}^{k}
$$

Следовательно, системой уравнений (5) функция $\varphi\left(x_{1}, \ldots, x_{n}\right)$ задана корректно, что и доказывает справедливость теоремы 1.

Нахождение верхних оценок параметра $h$ сопряжено с построением сравнительно сложных конструкций в $n$-мерной $k$-значной решетке $V_{n}^{k}$.

Выделим подмножество точек $\vec{x}=\left(x_{1}, \ldots, x_{n}\right)$ решетки $V_{n}^{k}$, в которое включим все такие точки $\vec{x}$, что

$$
\alpha_{i} \leq x_{i} \leq \beta_{i},
$$

где $\alpha_{i}<\beta_{i}, \quad \alpha_{i}, \beta_{i}, x_{i} \in\{0, \ldots, k-1\}, i=1, \ldots, n$.

Определение 2. Подмножество точек, удовлетворяющих условиям (7), будем называть доменом $D\left(d_{1}, d_{2}, \ldots, d_{n}\right)$ размерности $n$ и объема $d_{1} \times d_{2} \times \ldots \times d_{n}$, где $d_{i}=\beta_{i}-\alpha_{i}$.

Определение 3. Домен $D\left(d_{1}, d_{2}, \ldots, d_{n}\right)$ назовем внутренним, если в задающих его условиях (7) все нижние границы $\alpha_{i}$ отличны от $0\left(\alpha_{i} \neq 0\right.$ $i=1, \ldots, n)$ и все верхние границы $\beta_{j}$ отличны от $k-1\left(\beta_{j} \neq k-1, j=1, \ldots, n\right)$.

В геометрическом понимании домен образует некий $n$-мерный параллелепипед, целиком содержащийся в $V_{n}^{k}$. Если домен является внутренним, то ни одна его точка не лежит на гипергранях, образующих поверхность $V_{n}^{k}$.

Домен $D\left(d_{1}, d_{2}, \ldots, d_{n}\right)$, у которого все значения $d_{i} \geq 2$, назовем крупным.

Определение 4. Для внутреннего домена $D\left(d_{1}, d_{2}, \ldots, d_{n}\right)$, задаваемого условиями (7), домен $D\left(d_{1}+2, d_{2}+2, \ldots, d_{n}+2\right)$, определяемый условиями

$$
\alpha_{i}-1 \leq x_{i} \leq \beta_{i}+1, \quad i=1, \ldots, n,
$$

назовем окаймляющим. 
Заметим, что все точки исходного домена $D\left(d_{1}, d_{2}, \ldots, d_{n}\right)$ лежат внутри окаймляющего его домена $D\left(d_{1}+2, d_{2}+2, \ldots, d_{n}+2\right)$. Точки окаймляющего домена, не лежащие в исходном домене, будем называть граничными.

Определение 5. Функцию $k$-значной логики $g_{\alpha}\left(x_{1}, \ldots, x_{n}\right)$ назовем $\partial o$ менной, если она принимает значение $\alpha$ на точках, принадлежащих домену $D\left(d_{1}, d_{2}, \ldots, d_{n}\right)$, и только на них:

$$
g_{\alpha}\left(x_{1}, \ldots, x_{n}\right)=\alpha \Leftrightarrow\left(x_{1}, \ldots, x_{n}\right) \in D\left(d_{1}, d_{2}, \ldots d_{n}\right) .
$$

Теорема 2. Если домен $D\left(d_{1}, d_{2}, \ldots, d_{n}\right)$ - крупныий и внутренний, то пороговый индекс $r_{\alpha}$ уравнения, порожденного любой его доменной функиией

$$
g_{\alpha}\left(x_{1}, \ldots, x_{n}\right)=\alpha,
$$

равен $2 n$.

Доказательство. Пусть домен $D\left(d_{1}, d_{2}, \ldots, d_{n}\right)$ определятся неравенствами (7). Во-первых, убедимся, что уравнение (8) - выпуклое. Действительно, все точки, в которых оно выполняется, удовлетворяют условиям (7), содержащим ровно $2 n$ неравенств. Отсюда заключаем, что

$$
r_{\alpha} \leq 2 n \text {. }
$$

Остается доказать, что при выполнении указанных в формулировке леммы условий $r_{\alpha} \geq 2 n$. Для этого в множестве точек $V_{n}^{k} \backslash D\left(d_{1}, d_{2}, \ldots, d_{n}\right)$ выделим подмножество из $2 n$ точек:

$$
\begin{aligned}
\left(\alpha_{1}-1, \alpha_{2}+1, \alpha_{3}+1, \ldots, \alpha_{n}+1\right) & =\overrightarrow{\alpha_{1}}, \\
\left(\alpha_{1}+1, \alpha_{2}-1, \alpha_{3}+1, \ldots, \alpha_{n}+1\right) & =\overrightarrow{\alpha_{2}}, \\
\ldots & \ldots \quad \ldots \\
\left(\alpha_{1}+1, \alpha_{2}+1, \alpha_{3}+1, \ldots, \alpha_{n}-1\right) & =\overrightarrow{\alpha_{n}}, \\
\left(\beta_{1}+1, \beta_{2}-1, \beta_{3}-1, \ldots, \beta_{n}-1\right) & =\overrightarrow{\beta_{1}}, \\
\left(\beta_{1}-1, \beta_{2}+1, \beta_{3}-1, \ldots, \beta_{n}-1\right) & =\overrightarrow{\beta_{2}}, \\
\ldots \quad \ldots \quad \ldots & \\
\left(\beta_{1}-1, \beta_{2}-1, \beta_{3}-1, \ldots, \beta_{n}+1\right) & =\overrightarrow{\beta_{n}} .
\end{aligned}
$$

Докажем, что $\overrightarrow{\alpha_{1}}, \overrightarrow{\alpha_{2}}, \ldots, \overrightarrow{\alpha_{n}}, \overrightarrow{\beta_{1}}, \overrightarrow{\beta_{2}}, \ldots, \overrightarrow{\beta_{n}}-$ существенная система точек (см. [1]), т. е. что плоскость, задаваемая линейным неравенством и отсе- 
кающая полупространство точек $T \subseteq V_{n}^{k} \backslash D\left(d_{1}, d_{2}, \ldots, d_{n}\right)$, может отсечь не более одной точки из этой системы. Предположим противное: пусть подмножество $T$, названное пороговой конфигурацией точек (см. [1]), содержит две точки (или более) из системы (9). Сначала рассмотрим случай, когда $\overrightarrow{\alpha_{i}}, \overrightarrow{\alpha_{j}} \in T, \quad 1 \leq i<j \leq n$,

$$
\begin{aligned}
& \overrightarrow{\alpha_{i}}=\left(\alpha_{1}+1, \ldots, \alpha_{i-1}+1, \alpha_{i}-1, \alpha_{i+1}+1, \ldots, \alpha_{n}+1\right), \\
& \overrightarrow{\alpha_{j}}=\left(\alpha_{1}+1, \ldots, \alpha_{j-1}+1, \alpha_{j}-1, \alpha_{j+1}+1, \ldots, \alpha_{n}+1\right) .
\end{aligned}
$$

Пороговая конфигурация $T$ задается неравенством

$$
c_{1} x_{1}+\ldots+c_{n} x_{n} \geq c_{0}
$$

ему удовлетворяют все точки домена $D\left(d_{1}, d_{2}, \ldots, d_{n}\right)$, но, по нашему предположению, не удовлетворяют точки $\overrightarrow{\alpha_{i}}$ и $\overrightarrow{\alpha_{j}}$. Рассмотрим след, который плоскость $\wp$, задаваемая этим неравенством, оставляет на двумерной плоскости:

$$
\begin{gathered}
x_{1}=\alpha_{1}+1, \\
x_{2}=\alpha_{2}+1, \\
\ldots \\
x_{i-1}=\alpha_{i}+1, \\
x_{i+1}=\alpha_{i+1}+1, \\
\quad \ldots \\
x_{j-1}=\alpha_{j}+1, \\
x_{j+1}=\alpha_{j}+1, \\
\quad \ldots \\
x_{n}=\alpha_{n}+1 .
\end{gathered}
$$

Точки $\overrightarrow{\alpha_{i}}$ и $\overrightarrow{\alpha_{j}}$, очевидно, лежат на этой плоскости $\wp$ и имеют координаты $\left(x_{i}, x_{j}\right)$, равные $\left(\alpha_{i}-1, \alpha_{j}+1\right)$ и $\left(\alpha_{i}+1, \alpha_{j}-1\right)$ соответственно.

По предположению эти точки отсекаются, т. е. в них неравенство (10) не выполняется:

$$
\begin{gathered}
c_{1}\left(\alpha_{1}+1\right)+\ldots+c_{i-1}\left(\alpha_{i-1}+1\right)+c_{i}\left(\alpha_{i}-1\right)+ \\
+c_{i+1}\left(\alpha_{i+1}+1\right)+\ldots+c_{n}\left(\alpha_{n}+1\right)<c_{0}, \\
c_{1}\left(\alpha_{1}+1\right)+\ldots+c_{j-1}\left(\alpha_{j-1}+1\right)+c_{j}\left(\alpha_{j}-1\right)+ \\
\quad+c_{j+1}\left(\alpha_{j+1}+1\right)+\ldots+c_{n}\left(\alpha_{n}+1\right)<c_{0} .
\end{gathered}
$$


В то же время, как нетрудно проверить, точка $\overrightarrow{\alpha_{0}}=\left(\alpha_{1}+\right.$ $\left.+1, \ldots, \alpha_{i-1}+1, \alpha_{i}, \alpha_{i+1}+1, \ldots, \alpha_{j-1}+1, \alpha_{j}, \alpha_{j+1}+1, \ldots, \alpha_{n}+1\right)$ принадлежит домену $D\left(d_{1}, d_{2}, \ldots, d_{n}\right)$, лежит на плоскости ґ и имеет на ней координаты $\left(\alpha_{i}, \alpha_{j}\right)$. В этой точке неравенство (10) должно выполняться:

$$
c_{i} \alpha_{i}+c_{j} \alpha_{j} \geq c_{0}
$$

Складывая неравенства (11) и сопоставляя результат с (12), получаем противоречие, доказывающее, что любая пара точек $\overrightarrow{\alpha_{i}}, \overrightarrow{\alpha_{j}}-$ существенная. Аналогично доказывается, что $\overrightarrow{\beta_{i}}$ и $\overrightarrow{\beta_{j}}$ для любых $i, j \in\{1, \ldots, n\}-$ существенная пара точек.

Остается рассмотреть вариант, когда множеству $T$ принадлежат точка $\overrightarrow{\alpha_{i}}$ и точка $\overrightarrow{\beta_{j}}$ (пусть $\left.i<j\right)$ :

$$
\begin{aligned}
& \overrightarrow{\alpha_{i}}=\left(\alpha_{1}+1, \ldots, \alpha_{i-1}+1, \alpha_{i}-1, \alpha_{i+1}+1, \ldots, \alpha_{n}+1\right), \\
& \overrightarrow{\beta_{j}}=\left(\beta_{1}-1, \ldots, \beta_{j-1}-1, \beta_{j}+1, \beta_{j+1}-1, \ldots, \beta_{n}-1\right) .
\end{aligned}
$$

В этом случае простое доказательство путем проектирования на двумерную плоскость не проходит, однако логика доказательства сохраняется.

Пусть неравенство (10) эти две вершины отсекает, т. е. не выполняется при их подстановке:

$$
\begin{gathered}
c_{1}\left(\alpha_{1}+1\right)+\ldots+c_{i-1}\left(\alpha_{i-1}+1\right)+c_{i}\left(\alpha_{i}-1\right)+ \\
+c_{i+1}\left(\alpha_{i+1}+1\right)+\ldots+c_{n}\left(\alpha_{n}+1\right)<c_{0}, \\
c_{1}\left(\beta_{1}-1\right)+\ldots+c_{j-1}\left(\beta_{j-1}-1\right)+c_{j}\left(\beta_{j}+1\right)+ \\
+c_{j+1}\left(\beta_{j}-1\right)+\ldots+c_{n}\left(\beta_{n}-1\right)<c_{0} .
\end{gathered}
$$

В результате сложения неравенств (13) получаем:

$$
\begin{gathered}
c_{1}\left(\alpha_{1}+\beta_{1}\right)+\ldots+c_{i-1}\left(\alpha_{i-1}+\beta_{i-1}\right)+ \\
+c_{i}\left(\alpha_{i}+\beta_{i}-1\right)+c_{i+1}\left(\alpha_{i+1}+\beta_{i+1}\right)+\ldots \\
\ldots+c_{j-1}\left(\alpha_{j-1}+\beta_{j-1}\right)+c_{j}\left(\alpha_{j}+\beta_{j}+2\right)+ \\
+c_{j+1}\left(\alpha_{j+1}+\beta_{j+1}\right)+\ldots+c_{n}\left(\alpha_{n}+\beta_{n}\right)<2 c_{0} .
\end{gathered}
$$


Следующие две точки очевидно принадлежат домену $D\left(d_{1}, d_{2}, \ldots, d_{n}\right)$, так как $D\left(d_{1}, d_{2}, \ldots, d_{n}\right)$ - крупный домен:

$$
\begin{aligned}
& \vec{\alpha}^{\prime}=\left(\alpha_{1}, \alpha_{2}, \ldots, \alpha_{i-1}, \alpha_{i}, \alpha_{i+1}, \ldots, \alpha_{j-1}, \alpha_{j}+2, \alpha_{j+1}, \ldots, \alpha_{n}\right), \\
& \vec{\beta}^{\prime}=\left(\beta_{1}, \beta_{2}, \ldots, \beta_{i-1}, \beta_{i}-2, \beta_{i+1}, \ldots, \beta_{j-1}, \beta_{j}, \beta_{j+1}, \ldots, \beta_{n}\right)
\end{aligned}
$$

и в этих точках неравенство (10) должно выполняться:

$$
\begin{gathered}
c_{1} \alpha_{1}+c_{2} \alpha_{2}+\ldots+c_{i-1} \alpha_{i-1}+c_{i} \alpha_{i}+c_{i+1} \alpha_{i+1}+\ldots \\
\ldots+c_{j-1} \alpha_{j-1}+c_{j}\left(\alpha_{j}+2\right)+c_{j+1} \alpha_{j+1}+\ldots+c_{n} \alpha_{n} \geq c_{0}, \\
c_{1} \beta_{1}+c_{2} \beta_{2}+\ldots+c_{i-1} \beta_{i-1}+c_{i} \beta_{i}+c_{i+1} \beta_{i+1}+\ldots \\
\ldots+c_{j-1} \beta_{j-1}+c_{j}\left(\beta_{j}+2\right)+c_{j+1} \beta_{j+1}+\ldots+c_{n} \beta_{n} \geq c_{0} .
\end{gathered}
$$

Сложение неравенств (15)

$$
\begin{gathered}
c_{1}\left(\alpha_{1}+\beta_{1}\right)+\ldots+c_{i-1}\left(\alpha_{i-1}+\beta_{i-1}\right)+c_{i}\left(\alpha_{i}+\beta_{i}-1\right)+ \\
+c_{i+1}\left(\alpha_{i+1}+\beta_{i+1}\right)+\ldots \\
\ldots+c_{j-1}\left(\alpha_{j-1}+\beta_{j-1}\right)+c_{j}\left(\alpha_{j}+\beta_{j}+2\right)+c_{j+1}\left(\alpha_{j+1}+\beta_{j+1}\right)+\ldots \\
\ldots+c_{n}\left(\alpha_{n}+\beta_{n}\right) \geq 2 c_{0}
\end{gathered}
$$

приводит к противоречию с ранее полученным неравенством (14), что окончательно доказывает справедливость теоремы.

В заключение заметим, что если крупный домен не является внутренним, то в его задании $s$ границ (7) принимают свои крайние значения, $\alpha_{i}=0$ или $\beta_{j}=k-1$. Для такого домена, очевидно,

$$
r_{\alpha}=2 n-s, \quad s \leq n
$$

Домен $D\left(d_{1}, d_{2}, \ldots, d_{n}\right)$, не являющийся крупным, назовем каноническим при условии, что $d_{1}=d_{2}=\ldots=d_{n}=1$. Канонический домен с геометрической точки зрения представляет собой $n$-мерный единичный куб $D(1,1, \ldots, 1)$. Для такого домена условия теоремы 2 не выполняются, и задача минимизации числа неравенств, его задающих, оказывается значительно более сложной.

Если $D(1,1, \ldots, 1)-$ внутренний домен $(k \geq 4)$, то минимальное число неравенств, задающих его, очевидно не зависит от расположения домена внутри $V_{n}^{k}$. 
Поэтому для удобства дальнейшего рассмотрения домен $D(1,1, \ldots, 1)$ разместим в $V_{n}^{k}$ в соответствии с условиями

$$
1 \leq x_{i} \leq 2, i=1, \ldots, n \text {. }
$$

Точки этого внутреннего домена $D(1,1, \ldots, 1)$ будут окружены точками окаймляющего домена $D(3,3, \ldots, 3)$ с границами

$$
0 \leq x_{i} \leq 3, \quad i=1, \ldots, n \text {. }
$$

Очевидно, что система линейных неравенств

$$
c_{1}^{(j)} x_{1}+\ldots+c_{n}^{(j)} x_{n} \geq c_{0}^{(j)}, \quad j=1, \ldots, r,
$$

задающая канонический домен $D(1,1, \ldots, 1)$, будет отсекать все граничные точки окаймляющего домена $D(3,3, \ldots, 3)$.

Исходный канонический домен $D(1,1, \ldots, 1)$ и окаймляющий его домен $D(3,3, \ldots, 3)$ представляют собой $n$-мерные кубы со сторонами 1 и 3 соответственно. Для канонического домена $D(1,1, \ldots, 1)$ справедливо следующее утверждение, по существу вытекающее из доказательства теоремы 2.

Теорема 3. Если домен $D(1,1, \ldots, 1)$ - канонический и внутренний, то для порогового индекса уравнения, задаваемого его доменной функиией

$$
g_{D}\left(x_{1}, x_{2}, \ldots, x_{n}\right)=\alpha \Leftrightarrow\left(x_{1}, x_{2}, \ldots, x_{n}\right) \in D(1,1, \ldots, 1),
$$

справедлива оценка

$$
n \leq r_{\alpha} \leq 2 n .
$$

Доказательство, также не ограничивая общности рассмотрения, проведем для домена (18); из его задания вытекают выпуклость уравнения (20) и верхняя оценка порогового индекса.

Далее, по аналогии с доказательством теоремы 2, в множестве $V_{n}^{k} \backslash D(1,1, \ldots, 1)$ выделим подмножество точек, лежащих в окаймляющем домене

$$
\begin{gathered}
(0,2, \ldots, 2)=\overrightarrow{\alpha_{1}} \\
(2,0, \ldots, 2)=\overrightarrow{\alpha_{2}}, \\
\ldots \\
(2,2, \ldots, 0)=\overrightarrow{\alpha_{n}}
\end{gathered}
$$


и докажем, что (22) - существенная система точек. Предположим противное, полагая, что точки $\overrightarrow{\alpha_{i}}$ и $\overrightarrow{\alpha_{j}}$ при $i \neq j \quad(i<j)$ можно отсечь от точек домена $D(1,1, \ldots, 1)$ одним неравенством из системы (19)

$$
c_{1} x_{1}+\ldots+c_{n} x_{n} \geq c_{0},
$$

которое выполняется во всех точках домена и не выполняется одновременно и в $\overrightarrow{\alpha_{i}}$, и в $\overrightarrow{\alpha_{j}}$. Подставляя координаты $\overrightarrow{\alpha_{i}}$ и $\overrightarrow{\alpha_{j}}$ в это неравенство, получаем

$$
\begin{aligned}
& 2 c_{1}+\ldots+2 c_{i-1}+0 \cdot c_{i}+2 c_{i+1}+\ldots+2 c_{j-1}+2 c_{j}+2 c_{j+1}+\ldots+2 c_{n}<c_{0}, \\
& 2 c_{1}+\ldots+2 c_{i-1}+2 c_{i}+2 c_{i+1}+\ldots+2 c_{j-1}+0 \cdot c_{j}+2 c_{j+1}+\ldots+2 c_{n}<c_{0} .
\end{aligned}
$$

При этом точка $\quad \vec{\gamma}_{i, j}=(2, \ldots, 2,1,2, \ldots, 2,1,2, \ldots, 2) \quad$ принадлежит домену $D(1,1, \ldots, 1)$, и в ней неравенство (23) должно выполняться:

$$
2 c_{1}+\ldots+2 c_{i-1}+c_{i}+2 c_{i+1}+\ldots+2 c_{j-1}+c_{j}+2 c_{j+1}+\ldots+2 c_{n} \geq c_{0} .
$$

Складывая неравенства (24)

$$
4 c_{1}+\ldots+4 c_{i-1}+2 c_{i}+4 c_{i+1}+\ldots+4 c_{j-1}+2 c_{j}+4 c_{j+1}+\ldots+4 c_{n}<2 c_{0}
$$

и умножая на 2 неравенство (25)

$$
4 c_{1}+\ldots+4 c_{i-1}+2 c_{i}+4 c_{i+1}+\ldots+4 c_{j-1}+2 c_{j}+4 c_{j+1}+\ldots+4 c_{n} \geq 2 c_{0},
$$

получаем противоречие, доказывающее, что (22) - существенная система вершин. Отсюда вытекает, что

$$
r_{\alpha} \geq n
$$

Теорема доказана.

Уточнение оценки (21) потребует дополнительных усилий, направленных на изучение геометрических свойств канонического домена и особенностей расположения задающих его плоскостей.

Определение 6. Теневой гранью канонического домена $D(1,1, \ldots, 1)$, заданного условиями (18), назовем $(n-1)$-мерный домен, у которого одна из координат $x_{i}$ фиксирована: либо $x_{i}=0$, либо $x_{i}=3$, а остальные координаты $x_{j}$ удовлетворяют условиям

$$
1 \leq x_{j} \leq 2, j \neq i
$$


Теневую грань канонического домена при фиксации $x_{i}=0$ обозначим через $D\left(x_{i}=0\right)$, при $x_{i}=3$ - через $D\left(x_{i}=3\right)$. Все теневые грани лежат в окаймляющем домене (18): $D\left(x_{i}=0\right) \subset D(3,3, \ldots, 3) ; D\left(x_{i}=3\right) \subset D(3,3, \ldots, 3)$. Каждая теневая грань содержит $2^{n-1}$ точек. Теневые грани $D\left(x_{i}=0\right)$ и $D\left(x_{i}=3\right)$ будем называть противоположными.

В целом для канонического внутреннего домена (18) имеется $2 n$ теневых граней, состоящих из $n$ пар противоположных граней.

Пусть неравенство

$$
c_{1} x_{1}+\ldots+c_{n} x_{n} \geq c_{0}
$$

- одно из неравенств системы (19) - задает плоскость, которая отсекает (т. е. не имеет своими решениями) множество точек $T \subset V_{n}^{k}$, образующее пороговую конфигурацию точек (см. [1]).

Лемма 1. Пороговая конфигурачия Т из системы (19) не может иметь одновременно нетривиальные пересечения с двумя противоположными теневыми гранями.

Доказательство. Предположим противное:

$$
T \cap D\left(x_{i}=0\right) \neq \varnothing, \quad T \cap D\left(x_{i}=3\right) \neq \varnothing ;
$$

отсюда и из условия полной монотонности пороговой конфигурации (см. [1]) на плоскостях $x_{i}=0$ и $x_{i}=3$ решётки $V_{n}^{k}$ следует, что существует такая фиксация переменных

$$
\left(x_{1}, \ldots, x_{i-1}, x_{i+1}, \ldots, x_{n}\right)=\left(\xi_{1}, \ldots, \xi_{i-1}, \xi_{i+1}, \ldots, \xi_{n}\right),
$$

что пороговая конфигурация $T$ содержит точки $\left(\xi_{1}, \ldots, \xi_{i-1}, 0, \xi_{i+1}, \ldots, \xi_{n}\right)$ и $\left(\xi_{1}, \ldots, \xi_{i-1}, 3, \xi_{i+1}, \ldots, \xi_{n}\right), 1 \leq \xi_{v} \leq 2, v \neq i$.

В этих точках неравенство (26), задающее $T$, не выполняется:

$$
\begin{aligned}
& c_{1} \xi_{1}+\ldots+c_{i-1} \xi_{i-1}+c_{i} \cdot 0+c_{i+1} \xi_{i+1}+\ldots+c_{n} \xi_{n}<c_{0}, \\
& c_{1} \xi_{1}+\ldots+c_{i-1} \xi_{i-1}+c_{i} \cdot 3+c_{i+1} \xi_{i+1}+\ldots+c_{n} \xi_{n}<c_{0} .
\end{aligned}
$$

В то же время точки

$$
\left(\xi_{1}, \ldots, \xi_{i-1}, 1, \xi_{i+1}, \ldots, \xi_{n}\right) \text { и }\left(\xi_{1}, \ldots, \xi_{i-1}, 2, \xi_{i+1}, \ldots, \xi_{n}\right)
$$

принадлежат домену $D(1,1, \ldots, 1)$, в них неравенство (26) должно выполняться:

$$
\begin{aligned}
& c_{1} \xi_{1}+\ldots+c_{i-1} \xi_{i-1}+c_{i} \cdot 1+c_{i+1} \xi_{i+1}+\ldots+c_{n} \xi_{n} \geq c_{0}, \\
& c_{1} \xi_{1}+\ldots+c_{i-1} \xi_{i-1}+c_{i} \cdot 2+c_{i+1} \xi_{i+1}+\ldots+c_{n} \xi_{n} \geq c_{0} .
\end{aligned}
$$


Складывая попарно неравенства (27) и (28), получаем противоречие, доказывающее справедливость леммы.

Следовательно, пороговая конфигурация $T$ может иметь нетривиальные пересечения не более чем с $n$ теневыми гранями, среди которых нет противоположных. Поэтому будем без ограничения общности считать, что $T$ может иметь нетривиальные пересечения лишь с теневыми гранями

$$
D\left(x_{1}=0\right), D\left(x_{2}=0\right), \ldots, D\left(x_{n}=0\right) .
$$

Отметим, что если в теневой грани $D\left(x_{i_{1}}=0\right)$ рассмотреть фиксацию переменной $x_{i_{2}}, i_{1} \neq i_{2}$, например, $x_{i_{2}}=2$, то выделится подгрань теневой грани размерности $n-2$, которую обозначим $D\left(x_{i_{1}}=0, x_{i_{2}}=2\right)$. При фиксации последующих переменных будем получать подграни размерностей $n-3$ и т. д., при этом значения фиксируемых переменных $x_{i_{3}}=\varepsilon_{3}$ и т. д. будем указывать в скобках: $D\left(x_{i_{1}}=0, x_{i_{2}}=2, x_{i_{3}}=\varepsilon_{3}\right)$ и т. д.

Лемма 2. Пороговая конфигураиия $T$ из (19) не может одновременно иметь нетривиальные пересечения $c$ подгранями $D\left(x_{i}=0, x_{j}=2\right)$ $u D\left(x_{j}=0, x_{i}=2\right)$.

Доказательство. Без ограничения общности положим $i=1, j=2$. Предполагая противное, из условия полной монотонности пороговой конфигурации устанавливаем, что $T$ на подграни $D\left(x_{1}=0, x_{2}=2\right)$ содержит точку

$$
\left(0,2, \varepsilon_{3}, \ldots, \varepsilon_{n}\right) \in T
$$

и на подграни $D\left(x_{1}=2, x_{2}=0\right)-$ точку

$$
\left(2,0, \varepsilon_{3}, \ldots, \varepsilon_{n}\right) \in T
$$

для некоторой фиксации $\left(\varepsilon_{3}, \ldots, \varepsilon_{n}\right), 1 \leq \varepsilon_{i} \leq 2, i=3, \ldots, n$.

В указанных точках неравенство (20) не выполнено:

$$
\begin{aligned}
& c_{1} \cdot 0+c_{2} \cdot 2+c_{3} \varepsilon_{3}+\ldots+c_{n} \varepsilon_{n}<c_{0}, \\
& c_{1} \cdot 2+c_{2} \cdot 0+c_{3} \varepsilon_{3}+\ldots+c_{n} \varepsilon_{n}<c_{0},
\end{aligned}
$$

в то же время точка $\left(1,1, \varepsilon_{3}, \ldots, \varepsilon_{n}\right)$ принадлежит каноническому домену $D(1,1, \ldots, 1)$, и в ней неравенство (26) выполняется:

$$
c_{1} \cdot 1+c_{2} 1+c_{3} \varepsilon_{3}+\ldots+c_{n} \varepsilon_{n} \geq c_{0} .
$$


Складывая неравенства (29), получаем противоречие с неравенством (30), умноженным на 2, что и доказывает справедливость леммы.

Лемма 3. Пороговая конфигурачия $Т$ из (19) может пересекаться не более чем с $2^{n}-1$ точками теневых граней.

Доказательство. Из леммы 1 вытекает, что пороговая конфигурация $T$ может иметь нетривиальные пересечения не более чем с $n$ теневыми гранями, например, с $D\left(x_{1}=0\right), D\left(x_{2}=0\right), \ldots, D\left(x_{n}=0\right)$.

Также, без ограничения общности, можно считать, что

$$
\left|T \cap D\left(x_{1}=0\right)\right| \geq\left|T \cap D\left(x_{2}=0\right)\right| \geq \ldots \geq\left|T \cap D\left(x_{n}=0\right)\right| .
$$

Покажем, что при этом

$$
\left|T \cap D\left(x_{i}=0\right)\right| \leq 2^{n-i}
$$

Действительно, при $i=1$

$$
\left|T \cap D\left(x_{1}=0\right)\right| \leq\left|D\left(x_{1}=0\right)\right|=2^{n-1} .
$$

Если при $i=2$ окажется, что $\left|T \cap D\left(x_{2}=0\right)\right|>2^{n-2}$, то

$$
T \cap D\left(x_{2}=0, x_{1}=2\right) \neq \varnothing,
$$

так как

$$
D\left(x_{2}=0\right)=D\left(x_{2}=0, x_{1}=1\right) \cup D\left(x_{2}=0, x_{1}=2\right)
$$

и

$$
\left|D\left(x_{2}=0, x_{1}=1\right)\right|=\left|D\left(x_{2}=0, x_{1}=2\right)\right|=2^{n-2} .
$$

Отсюда, по лемме $2, T$ не может иметь нетривиального пересечения с $D\left(x_{1}=0, x_{2}=2\right)$, следовательно,

$$
\left|T \cap D\left(x_{1}=0\right)\right| \leq 2^{n-2},
$$

но тогда

$$
\left|T \cap D\left(x_{1}=0\right)\right|<\left|T \cap D\left(x_{2}=0\right)\right|,
$$

что противоречит условию (31). Поэтому

$$
\left|T \cap D\left(x_{2}=0\right)\right| \leq 2^{n-2}
$$

и, более того, все точки $T \cap D\left(x_{2}=0\right)$ лежат внутри подграни $D\left(x_{1}=0, x_{2}=0\right)$ размерности $n-2$. 
Далее, если при $i=3$ окажется, что

$$
\left|T \cap D\left(x_{3}=0\right)\right|>2^{n-3},
$$

то в силу полной монотонности $T$

$$
\begin{aligned}
& T \cap D\left(x_{3}=0\right)=T \cap D\left(x_{3}=0, x_{1}=1\right)=T \cap D\left(x_{3}=0, x_{1}=1, x_{2}=1\right) \cup \\
& \cup T \cap D\left(x_{3}=0, x_{1}=1, x_{2}=2\right)
\end{aligned}
$$

и

$$
T \cap D\left(x_{3}=0, x_{1}=1, x_{2}=2\right) \neq \varnothing
$$

или

$$
T \cap D\left(x_{3}=0, x_{2}=2\right) \neq \varnothing .
$$

Отсюда, также по лемме $2, T$ не может иметь нетривиального пересечения с $D\left(x_{3}=2, x_{2}=0\right)$, и

$$
\left|T \cap D\left(x_{2}=0\right)\right|<\left|T \cap D\left(x_{3}=0\right)\right|,
$$

что вновь противоречит условию (31).

Рассуждая аналогичным образом, по индукции, учитывая свойство полной монотонности пороговой конфигурации $T$, окончательно устанавливаем справедливость оценки (32). Отсюда для общей мощности пересечения пороговой конфигурации $T$ со всеми теневыми гранями получаем

$$
\begin{aligned}
& \left|T \cap D\left(x_{1}=0\right)\right|+\left|T \cap D\left(x_{2}=0\right)\right|+\ldots+\left|T \cap D\left(x_{n}=0\right)\right| \leq \\
& \leq 2^{n-1}+2^{n-2}+\ldots+2+1=2^{n}-1,
\end{aligned}
$$

что и требовалось доказать.

Теорема 4. Для порогового индекса $r_{\alpha}$ уравнения (20) справедлива оценка

$$
r_{\alpha} \geq n+1
$$

Доказательство. Из леммы 3 вытекает, что произвольная пороговая конфигурация $T$ может отсечь не более $2^{n}-1$ точек теневых граней окаймляющего домена. Всего теневых граней $2 n$, каждая содержит $2^{n-1}$ точек, 
и общее число точек теневых граней равно $n \cdot 2^{n}$. Отсюда следует, что $n$ пороговых конфигураций недостаточно для их отсечения, поэтому справедлива оценка (33).

Оценка (33) интересна тем, что для некоторых размерностей $n$ она дает точные значения порогового индекса $r_{\alpha}$. Покажем это путем непосредственного построения соответствующих минимальных систем неравенств при $n=2,3,4$.

1. При $n=2$ для канонического домена

$$
D(1,1)=\left\{\left(x_{1}, x_{2}\right): 1 \leq x_{1} \leq 2,1 \leq x_{2} \leq 2\right\}
$$

система из 3-х неравенств

$$
\left\{\begin{array}{l}
3 x_{1}-x_{2}>0 \\
3 x_{1}+x_{2}<9 \\
x_{2}>0
\end{array}\right.
$$

является минимальной, так как при $n=2$ оценка (33) имеет вид

$$
r_{\alpha} \geq 3 \text {. }
$$

2. При $n=3$ канонический домен

$$
D(1,1,1)=\left\{\left(x_{1}, x_{2}, x_{3}\right): 1 \leq x_{1} \leq 2,1 \leq x_{2} \leq 2,1 \leq x_{3} \leq 2\right\}
$$

задается системой из 4 неравенств

$$
\left\{\begin{array}{l}
3 x_{2}-x_{3}>0 \\
3 x_{2}+x_{3}<9 \\
3 x_{1}-x_{3}<6 \\
3 x_{1}+x_{3}>3
\end{array}\right.
$$

которая является минимальной, так как $r_{\alpha} \geq 4$.

3. При $n=4$ канонический домен, определённый неравенствами

$$
1 \leq x_{i} \leq 2, \quad i=1,2,3,4,
$$


можно представить системой неравенств

$$
\left\{\begin{array}{l}
9 x_{2}-3 x_{3}-2 x_{4}>-2 \\
9 x_{2}+3 x_{3}+2 x_{4}<29 \\
9 x_{1}-3 x_{3}+2 x_{4}<20 \\
9 x_{1}+3 x_{3}-2 x_{4}>7 \\
x_{4}>0
\end{array}\right.
$$

которая является минимальной, так как $r_{\alpha} \geq 5$.

На рис. 1, 2, 3 представлены канонические домены при $n=2,3,4$, лежащие внутри окаймляющих доменов. Точки канонических доменов выделены черным цветом. На каждом рисунке отмечены точки окаймляющих доменов, отсекаемые первым неравенством каждой из приведенных минимальных систем неравенств. Все прочие неравенства систем получены из первых с помощью линейного преобразования, за исключением неравенств $x_{2}>0$ при $n=2$ и $x_{4}>0$ при $n=4$.

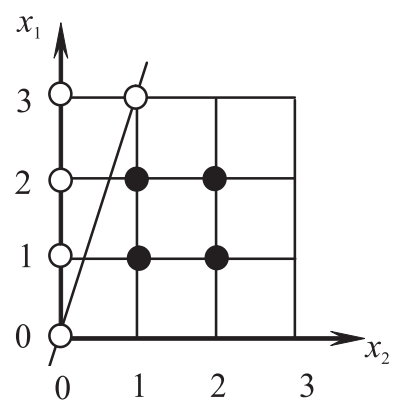

Рис. 1

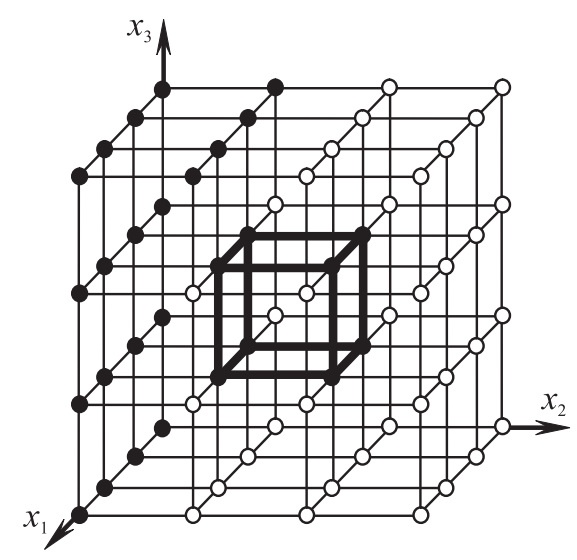

Рис. 2

Итак, при $n=2,3,4$ минимальное число неравенств, необходимое для задания канонического домена, точно равно

$$
r_{\alpha}=n+1
$$




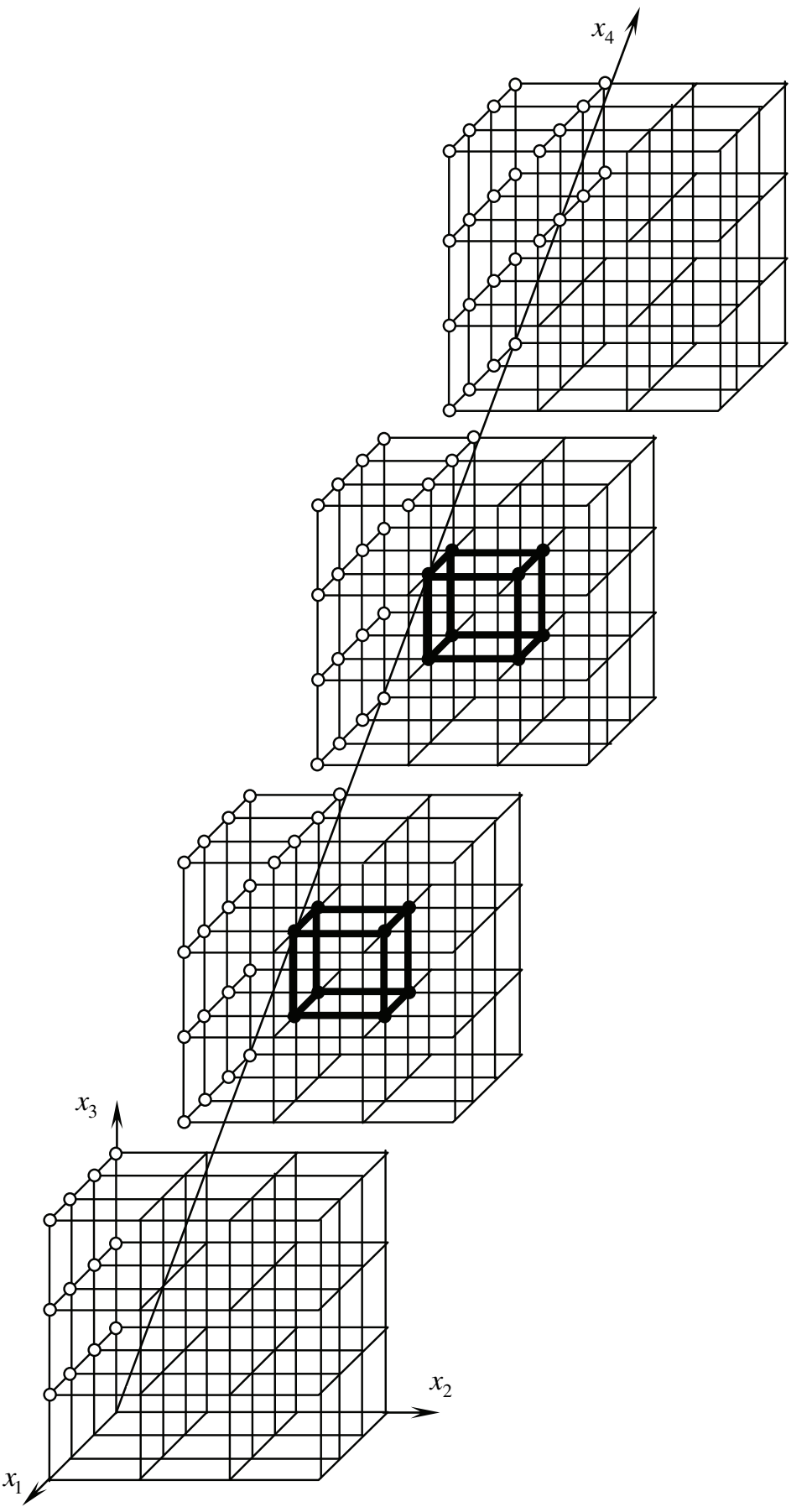

Рис. 3

2011, T. 2, № 1, C. 75-96 
Можно выдвинуть гипотезу о том, что и для $n \geq 5$ это равенство будет выполняться, однако поиск соответствующей минимальной системы уже при $n=4$ (и даже частично при $n=3$ ) сопряжен со сложными геометрическими построениями.

Приведем примеры вычисления пороговой сложности некоторых $k$-значных функций.

Теорема 5. При $k=m^{n}, m \geq 3$, существуют $k$-значные функции с суммарной пороговой сложностью

$$
h\left(m^{n}\right)=2 n\left(m^{n}-m^{n-1}\right) .
$$

Доказательство. Множество значений каждой переменной $X=$ $=\left\{0, \ldots, m^{n}-1\right\}$ разобьем на $m$ подмножеств

$$
\begin{aligned}
& X=X_{1} \cup X_{2} \cup \ldots \cup X_{m}, \\
& X_{1}=\left\{0, \ldots, m^{n-1}-1\right\}, \\
& X_{2}=\left\{m^{n-1}, \ldots, 2 m^{n-1}-1\right\}, \\
& X_{3}=\left\{2 m^{n-1}, \ldots, 3 m^{n-1}-1\right\}, \\
& \ldots \\
& X_{m}=\left\{(m-1) m^{n-1}, \ldots, m^{n}-1\right\} .
\end{aligned}
$$

Разбиение (38) приведет к разбиению всей $n$-мерной решетки $V_{n}^{k}$ на $m^{n}$ доменов

$$
X_{i_{1}, i_{2}, \ldots, i_{n}}=X_{i_{1}} \times X_{i_{2}} \times \ldots \times X_{i_{n}},
$$

так что точка $\vec{\xi}=\left(\xi_{1}, \xi_{2}, \ldots, \xi_{n}\right)$ принадлежит домену $X_{i_{1}, i_{2}, \ldots, i_{n}}$, если

$$
\xi_{1} \in X_{i_{1}}, \xi_{2} \in X_{i_{2}}, \ldots, \xi_{n} \in X_{i_{n}} .
$$

Зададим доменную функцию $f_{D}\left(x_{1}, \ldots, x_{n}\right)$, положив ее на всех точках каждого домена вида (39) равной одному значению

$$
f_{D}\left(x_{1}, \ldots, x_{n}\right)=\alpha_{i_{1}, i_{2}, \ldots, i_{n}} \Leftrightarrow\left(x_{1}, \ldots, x_{n}\right) \in X_{i_{1}, i_{2}, \ldots, i_{n}},
$$

а на точках разных доменов - разным значениям. Так как общее количество доменов равно $k=m^{n}$, то на каждом домене функция будет принимать одно из своих значений $0,1, \ldots, m^{n}-1$, и такое задание корректно. 
При $m \geq 3$ все построенные домены - крупные, поэтому по теореме 2 пороговый индекс $r_{\alpha}$ любого внутреннего крупного домена равен $r_{\alpha}=2 n$, а если домен не является внутренним и касается s границ решетки $V_{n}^{k}$ (см. (17)), то $r_{\alpha}=2 n-s$. При подсчете суммарного порогового индекса можно из числа $2 n \cdot m^{n}$ вычесть количество граней всех доменов, лежащих на поверхности $V_{n}^{k}$ как $n$-мерного куба, т. е. $2 n \cdot m^{n-1}$, отсюда и следует оценка (37).

Заметим, что уже при $k=9=3^{2}$ равенство (37) имеет вид

$$
h(9)=24 \text {, }
$$

в то время как для пороговой функции $h=2 \cdot 9-2=16$; при $k=27=3^{3}$ оценка (37) дает уже

$$
h(27)=108 \text {, }
$$

а для пороговой 27-значной функции $h=2 \cdot 27-2=52$.

Доказательство теоремы 5 основано на анализе функции, построенной с помощью крупных доменов. Однако доменные функции могут сочетать в своем строении домены различных размеров, в том числе крупные и канонические.

Теорема 6. При $k=12$ и $n=2$ существуют 12-значные функиии с суммарной пороговой сложностью

$$
h=32 \text {. }
$$

Доказательство построим на базе функции $\varphi\left(x_{1}, x_{2}\right)$, для построения которой решетку $V_{n}^{k}$ разобьем так, как это показано на рис. 4.

Изначально множество значений $X=\{0,1, \ldots, 12\}$ разобьем на 3 подмножества: $X_{1}=\{0,1,2,3\}, X_{2}=\{4,5,6,7\}, X_{3}=\{8,9,10,11\}$. Такое разбиение породит 9 крупных доменов $X_{i, j}=X_{i} \times X_{j}$,

$$
X_{i j} \in\left(\xi_{i}, \xi_{j}\right) \Leftrightarrow \xi_{i} \in X_{i}, \xi_{j} \in X_{j},
$$

и на всех этих доменах, за исключением домена $X_{22}$, положим $\varphi\left(x_{1}, x_{2}\right)$ равной одному из значений, соответственно, $\alpha_{0}, \ldots, \alpha_{7}$. Домен $X_{22}$, в свою очередь, разобьем на 4 канонических домена разбиением переменных $X_{2}=\{4,5,6,7\}$ на подмножества $X_{21}=\{4,5\}$ и $X_{22}=\{6,7\}$, т. е.

$$
X_{22}=X_{21} \times X_{21} \cup X_{21} \times X_{22} \cup X_{21} \times X_{21} \cup X_{22} \times X_{22} .
$$


На этих канонических доменах зададим функцию $\varphi\left(x_{1}, x_{2}\right)$ равной оставшимся значениям $\alpha_{8}, \alpha_{9}, \alpha_{10}, \alpha_{11}$ (см. рис. 4). Все эти канонические домены - внутренние, поэтому в суммарную пороговую сложность они, по теореме 4, внесут по 3 неравенства каждый. Остальные домены - крупные, но лежащие на границе решетки $V_{n}^{k}$, из них 4 требуют для своего задания по 3 неравенства, а 4, расположенные по углам решетки, — по 2 неравенства, отсюда

$$
h=3 \times 4+4 \times 3+4 \cdot 2=32 .
$$

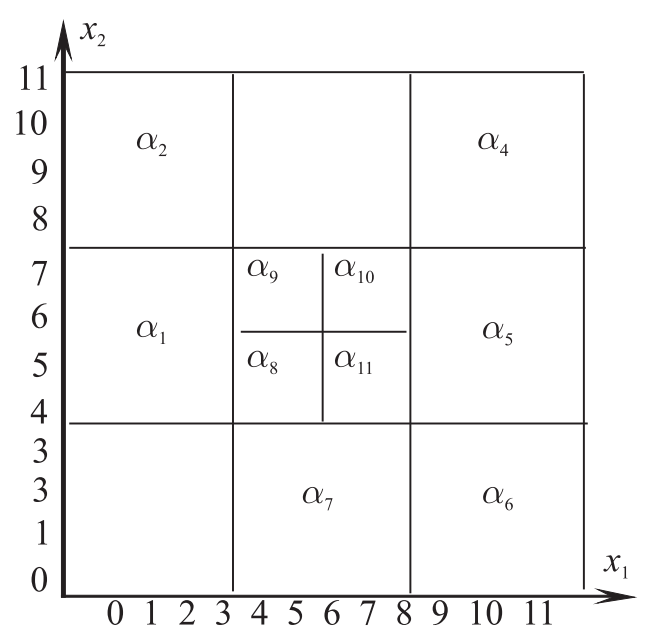

Рис. 4

Заметим, что оценка (40) существенно превышает аналогичную оценку при $k=12$ для пороговой функции, равную $h=22$.

В заключение подчеркнем, что поиск функций с максимальной суммарной пороговой сложностью при произвольных $k$ и $n$ в общем случае представляет собой сложную задачу дискретной математики. В то же время интересно отметить, что если в булевом случае максимальную сложность порогового представления имели линейные функции и близкие к ним (см. $[5,6,7])$, то, как показано в статье, среди $k$-значных функций высокая суммарная пороговая сложность обнаруживается у доменных функций, с геометрической точки зрения в определенном смысле аналогичных булевым линейным. 


\section{Список литературы}

1. Никонов В. Г., Никонов Н. В. Особенности пороговых представлений $k$-значных функций. - Труды по дискретной математике, т. 11 , вып. 1. - М.: ФИЗМАТЛИТ, 2008, с. 60-85.

2. Балакин Г. В., Никонов В. Г. Методы сведения булевых уравнений к системам пороговых соотношений. - Обозр. прикл. промышл. матем., 1994, т. 1, вып. 3, с. 389-401.

3. Никонов В. Г., Никонов Н. В. О применении методов пороговой логики для анализа и решения нелинейных булевых и $k$-значных уравнений. Материалы XXXII Междунар. конф. «Информационные технологии в науке, социологии, экономике и бизнесе, IT+S\&E'05», Запорожье: Запорож. нац. ун-т, 2005, с. 46-47.

4. Никонов Н. В., Рыбников К. К. Прикладные задачи, сводящиеся к анализу и решению систем линейных неравенств. Метод разделяющих плоскостей. - Вестник МГУЛ «Лесной вестник», 2002, № 2 (22), с. 191-195.

5. Никонов В. Г. Пороговые представления булевых функций. - Обозр. прикл. промышл. матем., 1994, т. 1, вып. 3, с. 402-457.

6. Никонов В. Г. Классификация минимальных базисных представлений всех булевых функций от четырех переменных. - Обозр. прикл. промышл. матем., 1994, т. 1, вып. 3, с. 458-545.

7. Никонов В. Г. Покрытия булевых графов. - Дискретн. матем., 1994, т. 6, вып. 4, с. 21-34.

8. Дертоузос М. Пороговая логика. — М.: Мир, 1967.

9. Бутаков E. A. Методы синтеза релейных устройств на пороговых элементах. - М.: Энергия, 1970.

10. Черников С. Н. Линейные неравенства. - М.: Наука, 1968.

11. Яблонский C. В. Введение в дискретную математику. - М.: Наука, 1986.

12. Яблонский С. В., Гаврилов Г. П., Кудрявиев В. Б. Функции алгебры логики и классы Поста. - М.: Наука, 1966. 

